\title{
A Rare Renal Cell Carcinoma Associated with Xp11.2 Translocation/TFE3 Gene Fusion: Case Report of 8 Cases and Review of the Literature
}

\author{
Mohammad Arman Hossain ${ }^{1,}$, , Jannatul Maoya Bashanti ${ }^{2,}$, Xiaoyong Dong ${ }^{1}$, Shun Gao ${ }^{1}$, \\ Hang Tong ${ }^{1}$, Xinyuan $\mathrm{Li}^{1}$, Hubin Yin ${ }^{1}$, Xin Gou ${ }^{1}$, Weiyang $\mathrm{He}^{1}$ \\ ${ }^{1}$ Department of Urology, The First Affiliated Hospital of Chongqing Medical University. Chongqing, China \\ ${ }^{2}$ Department of Imaging and Nuclear Medicine, The First Affiliated Hospital of Chongqing Medical University. Chongqing, China
}

Email address:

dr.armanhossain@yahoo.com (M. A. Hossain), jannatalin@yahoo.com (J. M. Bashanti)

${ }^{*}$ Corresponding author

\section{To cite this article:}

Mohammad Arman Hossain, Jannatul Maoya Bashanti, Xiaoyong Dong, Shun Gao, Hang Tong, Xinyuan Li, Hubin Yin, Xin Gou, Weiyang He. A Rare Renal Cell Carcinoma Associated with Xp11.2 Translocation/TFE3 Gene Fusion: Case Report of 8 Cases and Review of the Literature. International Journal of Clinical Urology. Vol. 5, No. 1, 2021, pp. 30-37. doi: 10.11648/j.ijcu.20210501.17

Received: April 4, 2021; Accepted: April 26, 2021; Published: May 8, 2021

\begin{abstract}
XP11.2 translocation TFE3 gene fusion renal cell carcinoma (RCC) is enlisted as a rare subtype of RCC by WHO in 2004. This type of Tumor was previously considered a pediatric tumor predominantly but is now invariably found in adult and older patients. The article investigates the clinical manifestation, pathological features, diagnosis criteria, and treatment of this rare subtype of RCC. We collected the clinical data of eight patients confirmed as XP11.2 TFE3 RCC admitted in the First affiliated hospital of Chongqing Medical University from August 2015 to November 2018, and a retrospective analysis of these data was conducted. There were three male, five female patients, the average age of 33.25 years old, ranging from 5 years old to 62 . Six patients were asymptomatic and diagnosed incidentally, one of them complained of hematuria and back pain for three months, and another patient complained just back pain for four months. After hospital admission, transabdominal ultrasonography, CT scan, and MRI imaging were done on all the patients. The tumor sizes of 2 patients were more than $7 \mathrm{~cm} ; 3$ patients were $5-6 \mathrm{~cm}$, and the rest three patients were below $4 \mathrm{~cm}$. One Patient got an Open Radical Nephrectomy for the right kidney and Right Ureterectomy. Four patients went through a Radical nephrectomy, and partial nephrectomies were done in the rest three patients. All the removed tumor samples were analyzed using pathology and immunohistochemistry assays. The results showed that all samples had renal carcinoma cells, with positive CD10 and TFE3 staining. Other parameters varied from each other. One postoperative Patient got both chemotherapies and targeted therapy; 3 patients were treated with Interferon but changed to thymopentin due to adverse effects. Only one Patient got thymopentin, and the rest three patients did not need any adjuvant therapy. Follow-up was done on all patients from 6 months to 40 months. Except for one patient who died of lung metastasis at 16 months after surgery, no disease progression occurred in those patients. The imaging findings are atypical, constantly varying among different patients with various tumor morphologies. So far, the golden standard of diagnosis is postoperative pathology and immune histochemistry. Radical or partial nephrectomy combining with renal lymph node clearance should be performed if metastasis of lymph nodes occurs. Proper adjuvant therapy, including chemotherapies, targeted therapy, and immune therapy, should be applied when needed. To maintain a risk-free life, follow-up must be done every 3 to 6 months.
\end{abstract}

Keywords: Renal Cell Carcinoma, XP11.2 Translocation, TFE3, Prognosis

\section{Introduction}

Renal cell carcinoma (RCC) originates within the lining of the proximal convoluted tubule, a neighborhood of the tiny tubes within the kidney. It is the most typical kidney cancer in adults, liable for around $90-95 \%$ of cases. RCC predominantly occurred in males over women with a ratio of $1.5: 1$. RCC mainly occurs between the sixth and seventh decennium of life. The prognosis is influenced by tumor size, histologic type, degree of invasion and metastasis, nuclear 
grade, etc. Staging is the most vital thing about the result of renal cell cancer. Overall, five years survival rate is $81 \%$, $74 \%, 53 \%$, and $8 \%$ for the Patient diagnosed in stage I, II, III, and IV, respectively. Renal cell carcinoma associated with Xp11.2 translocation with transcription factor E3 (TFE3) gene fusion is a sporadic tumor, and the prognosis of this Tumor is relatively poor than other subtypes of RCC. WHO classified it as a new subtype and distinct entity in 2004. [1] $\mathrm{Xp} 11.2 \mathrm{RCC}$ is characterized by translocations on chromosome Xp11.2, which results in gene fusion between TFE3 and other six possible partners. [7, 9] It is characterized by translocations involving the Xp11 breakpoint, which leads to gene fusions involving the TFE3 transcription factor gene, and maps to the present locus. [3] The most common subtypes of Xp11 translocation renal cell carcinomas are the ASPSCR1-TFE3 RCC resulting from a $t(X ; 17)(p 11 ; q 25)$ translocation [7] and the PRCC-TFE3 RCC, resulting from a $\mathrm{t}(\mathrm{X} ; 1)(\mathrm{p} 11 ; \mathrm{q} 21)$ translocation. This Tumor was primarily described in about $30 \%$ of the total RCC in a pediatric patient. However, recently Xp11.2/TFE3 RCC has been reported in the middle-aged adult population and older with metastasis in lymph nodes and poor prognosis. [2] Xp11.2 translocation RCC in adults could also be overlap with more common adult renal cell carcinoma subtypes, like and papillary cell and clear cell RCC. Here we report eight cases with Xp11.2 as well as a fusion in the TFE3 gene. There is a slight female predominance (F: $\mathrm{M}$ ratio, 1.6:1). Like other renal cell carcinomas, roughly one-third of all patients are asymptomatic, often accidentally discovered.

\section{Methods and Materials}

A total of eight patients were confirmedly diagnosed with XP11.2 TFE3 gene fusion RCC. There were three male and five female patients, with an average age of 33.25 years old, ranging from 5 years old to 62.6 patients were asymptomatic and diagnosed primarily incidentally. Furthermore, one complained of hematuria and back pain for three months, and another patient complained of just back pain for four months. After hospital admission, transabdominal ultrasonography, CT scan, and MRI were done on all the patients. The tumor sizes of 2 patients were more than $7 \mathrm{~cm} ; 3$ patients were $5-6$ $\mathrm{cm}$, and the rest three patients were below $4 \mathrm{~cm}$.

Right Open Radical Nephrectomy and Right Ureterectomy were performed for one Patient only. Four patients had undergone a Radical nephrectomy, and partial nephrectomies were done in the rest three patients.

All the tumor samples were sent for pathology and immunohistochemistry. All of them were positive for CD10, TFE3, and RCC. Other parameters varied from each other.

One Patient got both chemotherapies and targeted therapy postoperatively; 3 patients were treated with Interferon but changed to thymopentin because of adverse effects. One Patient got thymopentin only, and the rest three patients did not need any adjuvant therapy.

Follow-up was done to all patients from 6 months to the longest 40 months. Except for one patient, who died at 16 months of surgery due to lung metastasis, all patients were found normal without further disease progression.

We tried to search and find the related literature is in PubMed by using the keyword Renal cell carcinoma, TFE3, XP11.2 translocation and from there select the literature's related to XP11.2/TFE3 RCC patient's reported cases along with the presentation, diagnosis, and treatment, and other clinical morphologies and information and do a short review of those pieces of literature.

\section{Results}

This study was approved by the Urology Department in the First Affiliated Hospital of Chongqing Medical University, and all the participants gave their informed consent.

\subsection{Case 1}

A 14-year-old male with a history of hematuria associated with clots and right flank pain for three months. The abdominal contrast-enhanced CT scan was done, and it demonstrated irregular soft tissue in the right kidney area with a clear border, size: $7.6 \times 7.06 \times 6.86 \mathrm{~cm}$ (Figure 1). A slightly high and low-density tissue necrosis was found inside the Tumor. It reveals an uneven enhancement.
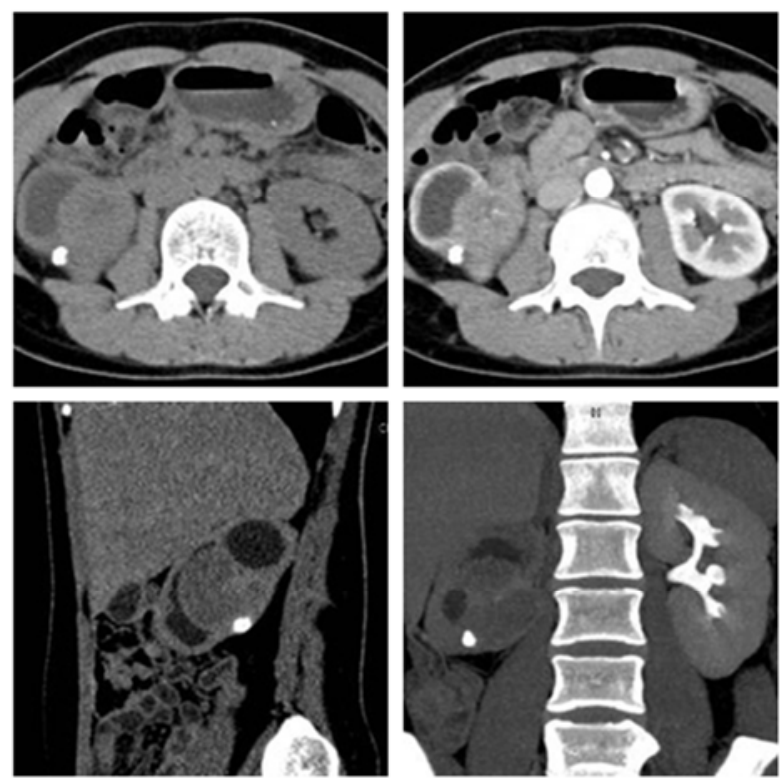

Figure 1. A mixed density Right renal tumor with a size of $7.6 \times 7.06 x$ $6.86 \mathrm{~cm}$.

Tumor attenuation (68 HU) was more significant than medulla $(28 \mathrm{HU})$ and renal parenchyma $(33 \mathrm{HU})$ during plain CT plain.

After appropriate evaluation under general anesthesia, the Patient underwent open retroperitoneal radical nephrectomy and perirenal fascia; perirenal fat was resected. Kidney, adrenal gland, and right Ureterectomy were done above the iliac blood vessel bifurcation and abdominal aorta. And inferior vena cava lymph node from the angle of the 
diaphragm to the bifurcation of the abdominal aorta (Figure 2).

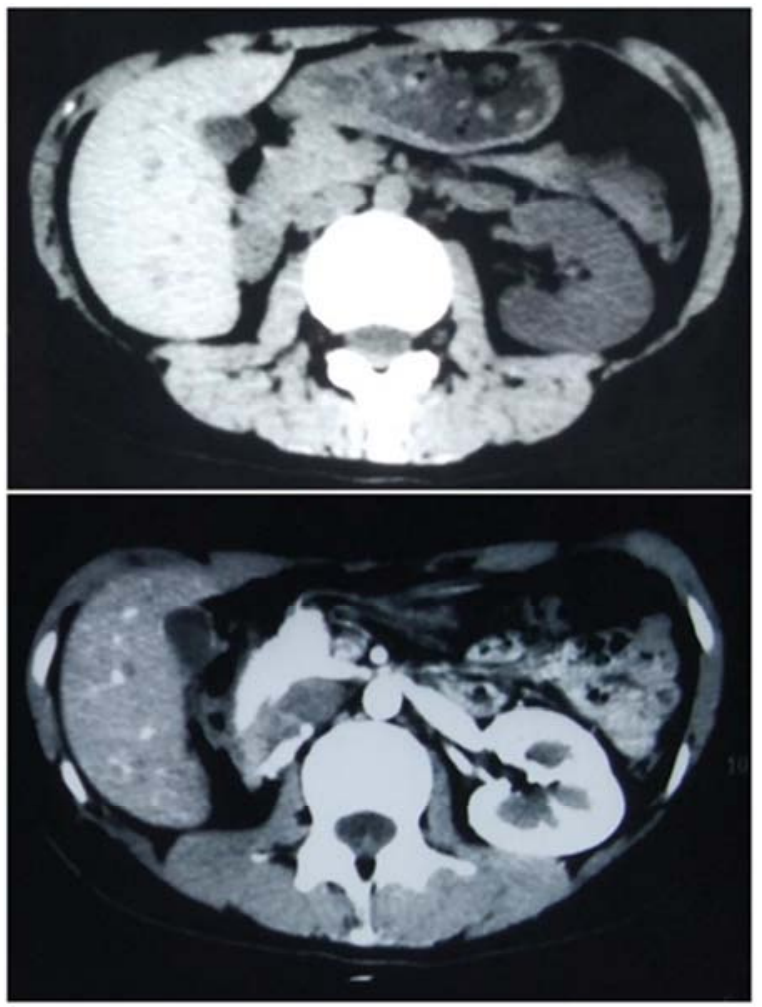

Figure 2. Postoperative CT scan and MRI after Right radical nephrectomy.

After the dissected right kidney's opening, rotten bleeding of gray-white colored Tumor of $7.2 \times 7 \times 7 \mathrm{~cm}$ was observed. Moreover, renal parenchyma was atrophied. Pathology revealed a case of XP11.2 translocation/TFE3 gene fusions associated with RCC with $7.2 \times 7 \times 7 \mathrm{~cm}$; the vascular region showed invasion of Tumor. The carcinoma metastases to the Left renal hilar lymph nodes as well. Immunohistochemistry results revealed positive for CK, EMA, CD-10, PAX-8, Vimentin, and TFE3. The Patient received no treatment after the operation. So, the Patient was discharged from the hospital after recovery. Nevertheless, sadly within six months after surgery, the patient did not come for re-examination. So, no adjuvant therapy was given within six months. After six months, Chest and abdominal computed tomography (CT) was done, and it shows patches were visible at the bottom of both lungs and were significantly enhanced. Irregular soft tissue mass shadows were observed in the right kidney area, with a clear boundary, about $2.9 \times 4.79 \times 2.62 \mathrm{~cm}$ in size, uneven internal density (42HU), and patchy high-density shadows were observed, which were enhanced unevenly. The patient was hospitalized. The Patient accepted chemical Therapy. Chemotherapy regimens: Vindesine $3.8 \mathrm{mg}$ at Day 1, Cyclophosphamide $1500 \mathrm{mg}$ at Day 1, Carboplatin $512 \mathrm{mg}$ at Day 3, and Etoposide 200mg on Day 4. After one month, he was examined again. Ultrasonography shows a hypoechoic mass with the size of $5.5 \times 4.5 \times 3.3 \mathrm{~cm}$ in the right kidney area, and CT shows diffuse lesions of both lungs. Right renal tumor is larger than before, $2.5 \times 4.82 \times 5.33 \mathrm{~cm}$. So, it was decided that Chemotherapy is not effective, and targeted therapy is recommended. The Patient began at Sunitinib $(4 * 50 \mathrm{mg}$ qid for four weeks and rest for two weeks). A repeat CT scan was done after one month, which shows the tumor size 2.59 × 4.86 $\mathrm{x} 3.7 \mathrm{~cm}$. The Patient ate the Sunitinib for four weeks, but because of hand-foot syndrome's side effect, the dose was changed to $3 * 37.5 \mathrm{mg}$ qid for four weeks and rest for two weeks. Repeated USG was done after two months, and it shows tumor size $3.5 \times 2.6 \times 1.7 \mathrm{~cm}$. The Patient continued Sunitinib for three more months, then developed fever and pain in the right flank area and admitted to the hospital.

Moreover, because of his economic factor, drugs changed from Sunitinib to Sorafenib. Furthermore, after five months of continuation of Sorafenib Patient died due to Lung Metastasis. A total of 16 months of follow-up from surgery to death were done.

\subsection{Case 2}

A 62-year-old male was symptom-free, had undergone a urinary occult blood test, which was positive, and a simple CT scan was done. The result shows Right renal mass, size $7.7 \mathrm{~cm}$ x $6 \mathrm{~cm}$. He underwent Laparoscopic Right Radical Nephrectomy. Postoperative pathology revealed Xp11.2translocations/ TFE3 gene fusions associated with RCC. Immunohistochemical results showed positive for Ki67 is $5 \%, \mathrm{CK}, \mathrm{SMA}, \mathrm{CD} 10$, Vimentin, Melan-A, RCC, and TFE3. AJCC staging was pT1bN0M0. Postoperatively he received Interferon $\alpha 2 b$ therapy for half a year ( 1 branch qid) and discontinued due to adverse reactions. Then he was on immunotherapy, Thymopentin, for half a year (10mg qid). We followed up with him for 30 months. CT was reviewed each 6-month within two years after surgery, which showed no evidence of recurrence or metastasis. The Chest CT showed small pulmonary nodules, but there was no chance of it in our follow-up the whole time.

\subsection{Case 3}

A 42-year-old healthy male was asymptomatic before and diagnosed incidentally with Left renal mass. He underwent with middle abdomen plain CT scan, and it shows a $5.5 \mathrm{~cm} \mathrm{x}$ $4.2 \mathrm{~cm}$ round tumor with a clear boundary. Laparoscopic left Radical Nephrectomy was done and finally diagnosed as XP11.2 RCC. Immunohistochemical results showed positive for CAIX- cell ring positive, TFE-3, CD10, RCC, Pax-8 partially favorable, Ki67, and Vimentin positive. AJCC staging was pT1bN0M0. He received no Adjuvant therapy. We followed up with him for 14 months (CT was reviewed in each 6-month) and showed no recurrence or metastasis.

\subsection{Case 4}

A 36-year-old healthy female was asymptomatic before and diagnosed incidentally with Left renal mass. She underwent with middle abdomen plain CT scan, and it shows around Tumor, the size was $5 \mathrm{~cm} \times 4 \mathrm{~cm}$ round tumor with a clear boundary. Laparoscopic left Partial Nephrectomy was performed and finally diagnosed as XP11.2 RCC. Immunohistochemical results showed positive for CAIX, TFE-3, RCC, CD10, Pax-2, and 
Vimentin. AJCC staging was pT1bN0M0. She was given Interferon $\alpha 2 b$ therapy for six months but discontinued due to adverse reactions, and then was received Thymopentin therapy for two years. She was followed up for 3 years (the 6-month/18month/30-month: transabdominal USG, 12-month/24-month 136-month: CT/ chest X-ray). She showed no evidence of recurrence or metastasis.

\subsection{Case 5}

A 5-years-old relatively healthy female child was asymptomatic and incidentally diagnosed with right renal mass CECT shows a $3.5 \times 4 \mathrm{~cm}$ mass in her right kidney, regular shape. Then she was admitted to the hospital and underwent a Laparoscopic right partial nephrectomy. The Tumor was considered as translocation of XP11.2, which is also associated with pigment differentiation and low malignant characteristics. Immunohistochemistry revealed a favorable rate for TFE3, RCC, CD10, PAX-8, Vimentin, and Ki67 is 5-10\%. CK and E-Cadherin were partially positive. Her AJCC staging was pT1aN0M0. After 12 months of her surgery, the patient did not present with any recurrence or metastasis. CT was reviewed each 6-month. So, did not need any further treatment.

\subsection{Case 6}

A 52-years-old female patient presented with lumbago for four months. A USG scan was done to reveal the cause and diagnosed with a Left renal mass of $3.9 \times 5 \mathrm{~cm}$ in size. The Patient went through laparoscopic left partial nephrectomy and was finally diagnosed as an XP11.2 TFE3 RCC. AJCC staging was pT1aN0M0. Her immunohistochemistry reports were positive for CD10, PAX-2, PAX-8, Vimentin, Ki67 was $80 \%$, E-cadherin, P504s, E-Cad, RCC partially, and TFE3. She was given Thymopentin therapy for a half month and followed up for ten months (CT was reviewed after 6month), showed no evidence of recurrence or metastasis.

\subsection{Case 7}

A 23-year-old healthy female was asymptomatic before and diagnosed incidentally with a Right renal mass. She underwent with plain CT scan, and it shows around Tumor, the size was $5.3 \times 4.2 \mathrm{~cm}$ round Tumor with a clear boundary.
AJCC staging was pT1bNxM0. Laparoscopic Right Partial Nephrectomy was done and finally diagnosed as ASPL-TFE3 RCC. Immunohistochemical results showed positive for CAIX, TFE-3, RCC, CD10, Pax-8, and P504s. She was given no therapy after the surgery. Hence followed up for six months (CT was reviewed in 6-month). She showed no evidence of recurrence or metastasis.

\subsection{Case 8}

A 32-year-old female was symptom-free and incidentally diagnosed with a Right renal Tumor, size $2.3 \times 3 \mathrm{~cm}$. She underwent a Laparoscopic Right partial Nephrectomy. Postoperative pathology revealed Xp11.2translocations/TFE3 gene fusions associated with RCC. Immunohistochemical results showed positive for Pax-8, CD10, Vimentin, P504s, RCC, and TFE3. Her AJCC staging was pT1aN0M0. She was given Interferon $\alpha 2 b$ therapy for two months (1 branch qid); and Thymopentin therapy for two months (10mg qid). She was followed up for six months (USG/ chest X-ray was reviewed in 6-month) and showed no recurrence or metastasis.

\section{Discussion}

An adult patient with XP11.2 Translocation Renal Cell Carcinoma with a large tumor and in the advanced stage has a relatively poor prognosis than the pediatric Patient. RCC associated with XP11.2/TFE3 gene fusion is a rare tumor that is primarily found in children. [3] Ellati et al. first concluded it as a rare tumor. [4] If we do the calculation, this TFE3 RCC occurs in only $1-1.6 \%$ of the adult patient, whereas it occurs $20-40 \%$ in children and young patients with total renal cell carcinoma. [5] Argani et al. reported, XP11.2 renal cell carcinoma usually occurred from TFE3 gene fusion with one of five different genes, which includes ASPL (17q25), PRCC (1q21), PSF (1q34), NonO (Xq12), and CLTC (17q23). [6, 7] According to Mir et al., Renal Cell Carcinoma, which is not classified, may have adverse clinicopathological parameters associated with positive expression of TFE3. They also reported that up to 5 years survival rate is $15.6 \%$ in TFE3-positive patients compared to $87.5 \%$ rate for TFE3-negative patients. [8]

Table 1. The tabulated form of general information about our total of eight patients.

\begin{tabular}{|c|c|c|c|c|c|c|c|c|}
\hline Case & $\begin{array}{l}\text { Age }(y r) / \\
\text { Sex/Laterality }\end{array}$ & Symptom & $\begin{array}{l}\text { Tumor } \\
\text { size }(\mathrm{cm})\end{array}$ & Operation & $\begin{array}{l}\text { Final } \\
\text { diagnosis }\end{array}$ & AJCC stage & Adjuvant Therapy & $\begin{array}{l}\text { Follow-up } \\
\text { (months) and } \\
\text { outcome }\end{array}$ \\
\hline 1 & 14/M/Rt & $\begin{array}{l}\text { Hematuria and } \\
\text { backache ( } 3 \text { months) }\end{array}$ & $\begin{array}{l}7.6 \times 7.06 \\
\times 6.86\end{array}$ & $\begin{array}{l}\text { ORN + right } \\
\text { Ureterectomy }\end{array}$ & Xp11.2 RCC & pT1bNxM0 & $\begin{array}{l}\text { Chemical \&Targeted } \\
\text { therapy }\end{array}$ & $\begin{array}{l}\text { 16, Died of lung } \\
\text { metastasis }\end{array}$ \\
\hline 2 & $62 / \mathrm{M} / \mathrm{Rt}$ & Asymptomatic & $7.7 \times 6$ & LRN & Xp11.2 RCC & pT1bN0M0 & $\begin{array}{l}\text { Interferon \& } \\
\text { Thymopentin }\end{array}$ & 30 , Normal \\
\hline 3 & $42 / \mathrm{M} / \mathrm{Lt}$ & Asymptomatic & $5.5 \times 4.2$ & LRN & Xp11.2 RCC & pT1bN0M0 & No therapy & 14, Normal \\
\hline 4 & $36 / \mathrm{F} / \mathrm{Lt}$ & Asymptomatic & $5 \times 4$ & LPN & Xp11.2 RCC & pT1bN0M0 & $\begin{array}{l}\text { Interferon \& } \\
\text { Thymopentin }\end{array}$ & 40, Normal \\
\hline 5 & $5 / \mathrm{F} / \mathrm{Rt}$ & Asymptomatic & $3.5 \times 4$ & LRN & $\mathrm{Xp11.2} \mathrm{RCC}$ & pT1aN0M0 & No therapy & 12, Normal \\
\hline 6 & $52 / \mathrm{F} / \mathrm{Lt}$ & Backache (4months) & $3.9 \times 5$ & LPN & Xp11.2 RCC & pT1aN0M0 & Thymopentin & 10 , Normal \\
\hline 7 & $23 / \mathrm{F} / \mathrm{Rt}$ & Asymptomatic & $5.3 \times 4.2$ & LRN & Xp11.2 RCC & pT1bN0M0 & No therapy & 6, Normal \\
\hline 8 & $32 / \mathrm{F} / \mathrm{Rt}$ & Asymptomatic & $2.3 \times 3$ & LPN & Xp11.2 RCC & pT1aN0M0 & $\begin{array}{l}\text { Interferon \& } \\
\text { Thymopentin }\end{array}$ & 6 , Normal \\
\hline
\end{tabular}


In our study, most of the Patient was asymptomatic and diagnosed incidentally. The main clinical features of this subtype of renal cell carcinoma are hematuria and low back pain. One Patient had both the symptom, and another patient had lumbago only. Some past study says morphology of XP11.2 TFE3 Tumor is different from other clear cells renal cell carcinoma. [9]

On CT scan or MRI imaging, RCC shows its features. Kato et al. [10] summarized that because of morphological similarity between this XP11.2 TFE3 renal cell tumor and papillary renal cell carcinoma, imaging findings are mostly the same. Some scholars already concluded with the CT and MRI report of 20 patients. They reported that tumor depletion in a plain CT scan in many cases is more potent than that in kidney parenchyma. However, at the time of enhanced phases, tumor enhancement remains less potent than the renal cortex but more potent than the medulla. [11] In the late phase, the Tumor was less intense than the medulla. On MRI scan, cancer remained unchanged intensity in T1W1, low signal intensity in $\mathrm{T} 2 \mathrm{~W} 1$ was not the same and relatively high signal intensity in DWI. Liu et al. [12] presented CT and MRI scan reports of five patients. In where MRI scan report of four patients present with moderate intensity, relatively higher signal intensity on T1 weighted MRI, three patient present low signal intensity on T2 Imaging, and the rest of the case present a higher signal intensity because of the bleeding from the Tumor. Computed tomography (CT) scan of two patients preset relatively higher tumor density than the renal cortex. [12] CT scan result of our 8 cases varies from one to another and not match up with the above discussion. Our first patient tumor depletion was higher than the kidney parenchyma and medulla during plain CT scan (Figure 1). Without adding the bleeding part, the depletion was relatively less intense than kidney parenchyma and medulla in arterial and venous phases. Among the cases that we have present earlier, only the first Patient's Tumor was in an advanced stage and very aggressive, which had necrosis and hemorrhage inside the Tumor. So, the imaging features are also different than the rest of the cases. If we compare all the reported cases reported previously, the only features in a simple CT scan of an XP11.2 RCC may be a solid mass only, and their attenuation was higher than the kidney parenchyma and medulla as well. [32-34] This is in the arterial phase, but it might be weaker in the renal cortex and medulla in the venous phase. Whatever the enhancement was always varied on the Tumor condition and the presence of hemorrhage and necrosis inside the Tumor, which usually occurs in an advanced stage or a very aggressive stage related to the age and sex of the Patient. However, more cases and reporting are required to conclude diagnostic criteria of an XP11.2 TFE3 gene fusion tumor by CT scan and MRI imaging. XP11.2 TFE3 gene fusion renal cell carcinoma shows various pathological characteristics similar to Clear cell Renal cell carcinoma and papillary renal cell carcinoma. [13] The typical morphology of XP11.2 RCC exhibits a nested or papillary manner, with lots of volume of clear or eosinophilic cytoplasm (Figure 3 ).

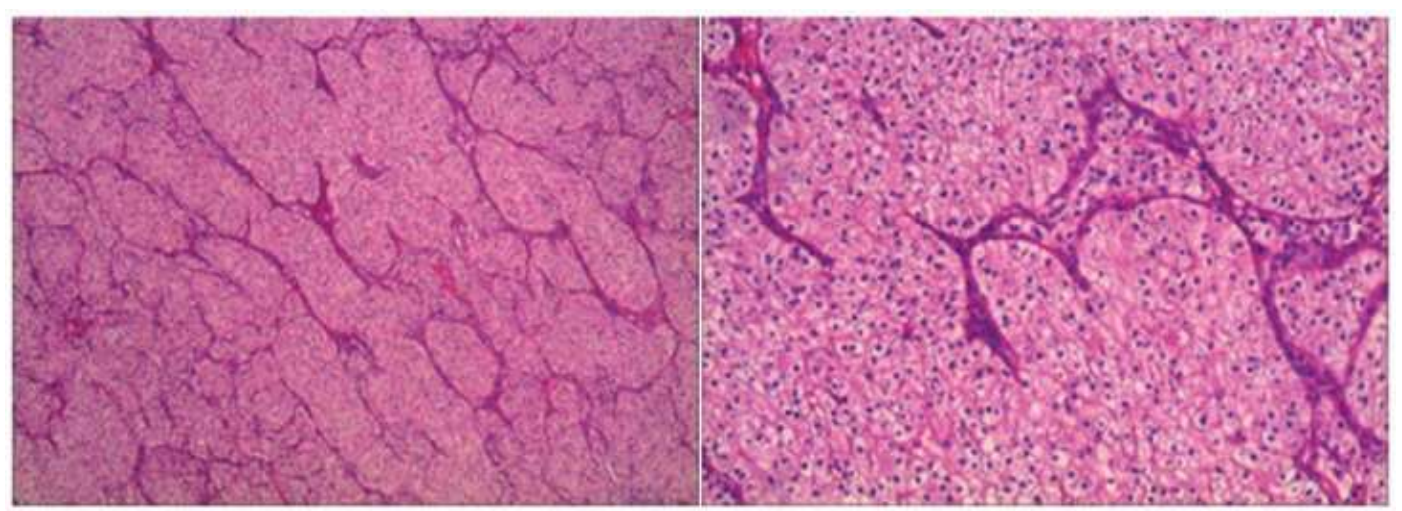

Figure 3. Some tumor cells were arranged in nests and had clear cytoplasm. And the others were arranged in papillary formations. The cytoplasm was eosinophilic. The image was taken in Hematoxylin and eosin staining. Magnification, the left panel was $x 40$, and the right panel was $x 200$.

As Renal cell carcinoma is associated with XP11.2 translocation, TFE3 gene fusion still does not have any specific Imaging diagnostic criteria yet, and the diagnosis is mainly conducted by pathological and immunohistochemical examination. The diagnosis of XP11.2 translocation renal cell carcinoma is mainly made on suspected base on clinical information, histological, and immunohistochemical features. Nevertheless, detecting chromosome translocation may be confirmed, which involves TFE3 gene fusion at the Xp11.2 chromosome. [28] We can lookout for the papillary cell arrangement, eosinophilic, hyaline nodules, abundant cytoplasm, and psammoma bodies. [27] Nuclear immunoreactivity for TFE3 protein by routine immunohistochemistry is a highly sensitive and specific marker. [29, 30] Cases discussed in previous literature do have some same immunohistochemical findings. In all the cases CD10 and TFE3 are positive. Meyer et al. [14] reported 5 cases wherein immunohistochemistry result Vimentin was positive in all the cases but SMA, CD45, and HMB45 were negative. Armah et al. [15] reported a case where AE1/AE3, CK7, EMA, RCA, CAIX all were positive. Ahluwalia et al. [16] reported 2 cases where CD10, Vimentin, and EMA were positive, but CK7 was negative. 
Table 2. The Immunostaining profiles of 8 patients.

\begin{tabular}{|c|c|c|c|c|c|c|c|c|}
\hline & Patient1 & Patient2 & Patient3 & Patient4 & Patient 5 & Patient6 & Patient 7 & Patient8 \\
\hline CAIX & + & - & Cell ring+ & - & - & - & + & - \\
\hline TFE3 & + & + & + & + & + & + & + & + \\
\hline $\mathrm{RCC}$ & + & Part+ & + & + & + & Part+ & + & + \\
\hline CD10 & + & + & + & + & + & + & + & + \\
\hline PAX-2 & + & - & - & - & - & + & - & - \\
\hline PAX-8 & - & - & Part+ & + & + & + & + & + \\
\hline VIM & + & + & + & Part+ & + & + & - & Part+ \\
\hline CK7 & - & - & - & - & - & - & - & - \\
\hline KI67 & $3 \%$ & $5 \%$ & + & - & $5-10 \%$ & $80 \%$ & $8-10 \%$ & - \\
\hline CK & - & + & - & + & Part+ & - & - & - \\
\hline MELANA & - & + & - & - & - & - & - & - \\
\hline SMA & - & + & - & - & - & + & - & - \\
\hline E-Caherine & - & - & - & - & + & + & - & - \\
\hline P504s & - & - & - & - & - & - & + & + \\
\hline
\end{tabular}

In the above Table 2, we tabulated the immunostaining profile of our eight patients. Here we can see that the immunohistochemical results are not consistent in all eight patients. Only TFE3, RCC, and CD10 are positive in all patients. Moreover, except for patient no-7, Vimentin was also positive in all the cases. PAX 8 was positive for six patients, where PAX 2 was only positive for two patients. E Caherine was positive for patients no. 5 and 6 only, whereas P504s was only positive for patients no. 7 and 8. After analyzing the data, we can say that only TFE3, CD10, and Vimentin may help diagnose XP11.2 TFE3 Renal cell carcinoma, but it may also be associated with age, sex, tumor size, and staging. However, using FISH to identify the gene of this rare Tumor may be an essential method. [16, 35] Some recent studies also showed that Immunohistochemistry for TFE3 might also be associated with a high false-positive result rate. [17] So much more valuable data is still required in this regard.

For choosing the treatment option in XP11.2 TFE3 renal cell carcinoma, the first choice should be radical or partial nephrectomy and hilar lymph nodes' clearance, if needed. [18] Though we do partial nephrectomy to our three patients. However, it also depends on the size and the nature of the Tumor. If the tumor size is less than $7 \mathrm{~cm}$, nephron-sparing surgery may consider as a treatment option. [19] However, there have been no established references for the treatment of this rare Tumor to date. Immunotherapy like interleukin-2 and interferon-alpha seems to be beneficial in some patients. According to Kakoki et al. [20], rapamycin inhibitors may be effective for XP11.2 TFE3 renal cell carcinoma as well. Oliver et al. [31] reported a case of 44 years old Patient treated with an everolimus (mTOR) inhibitor for an Xp11.2 translocation/TFE3 gene-fusion RCC after two previous failed treatments with a tyrosine kinase inhibitor, and the Patient obtained a long-lasting response of 25 months without any symptom. Some targeted therapy like Sunitinib, Sorafenib can also be applied. [21] Choueiri et al. reported 15 cases, where ten patients received Sunitinib, three patients received sorafenib, and two patients received monoclonal anti-VEGF antibody. [22] Three cases had somewhat response; in seven patients, the disease was stable, and in five patients, the disease progressed. In our reported cases, three patients got Interferon, and because they developed some adverse effects, the drug was changed to Thymopentin. Moreover, all four patients responded very well to this immunotherapy up to 40 months, 30 months, ten months, and six months follow-up, respectively, of no recurrence or complaint. 3 Patients did not receive any adjuvant therapy and went through 14, 12, and 5 months follow-up, respectively, maintaining the everyday lifestyle. Only our Patient no. 1, where the Tumor was very aggressive and extensive, even after receiving Chemotherapy, targeted therapy (Sunitinib and sorafenib) postoperatively, cancer metastasis to lungs, and survived for 16 months.

Though XP11.2 TFE3 renal cell carcinoma was previously considered that, it mainly occurs in children and of $20-40 \%$ of pediatric renal cell carcinoma and $1-1.6 \%$ of RCCS in adults $[23,24]$ now, it becomes more common in adult also associated with very aggressive in nature and poor prognosis. [25] Klatte et al. reported that an adult patient's mean survival rate is up to two years, where it is 6.3 years in the pediatric Patient. [26]

\section{Conclusion}

From observing our eight patients and all the references of XP11.2 Translocation factor E3 Renal cell carcinoma, we can summarize that Firstly, Immunohistochemistry may be the confirmatory criteria for diagnosing XP11.2 TFE3 RCC rather than Imaging. Secondly, for well-margined localized XP11.2 Tumor radical or partial nephrectomy and, if needed, renal hilar lymph node clearance is essential as a treatment for this type of RCC. Thirdly immunomodulating drug is more beneficial for treating the Patient with XP11.2 TFE3 renal cell carcinoma, rather than Chemotherapy or targeted therapy. VEGF - targeted therapy showed some efficacy in adult patients of XP11.2 RCC with lymph node or organ metastasis. Fourthly lifelong follow-up every three or six months with the physical examination, laboratory test, Chest, and abdominal Imaging is necessary. However, much more clinical data is needed to set up a parameter for the detection, treatment, immunization, and follow-up criteria of XP11.2 
TFE3 Renal cell tumor.

\section{Conflicts of Interest}

The authors do not declare any conflict of interest.

\section{Guarantor}

Mohammad Arman Hossain and Jannatul Maoya Bashanti had full access to all the data in the study and took responsibility for the data integrity and accuracy of the data analysis.

\section{Contributorship}

Mohammad Arman Hossain, Jannatul Maoya Bashanti, and Xiaoyong Dong did the data collection and wrote the first draft of the manuscript. All authors reviewed and edited the manuscript and approved the final version of the manuscript.

\section{Funding}

Any funding did not support this work.

\section{Acknowledgements}

We would like to thank Professor He Weiyang for his assistance and guidance in this paper.

\section{References}

[1] Lopez-Beltran A, Scarpelli M, Montironi R, et al: 2004 WHO classification of the renal tumors of the adults. Eur Urol 2006; 49 (5): 798-805.

[2] P. Argani and M. Ladanyi, et al.: Translocation carcinomas of the kidney. Clinics in Laboratory Medicine, 2005; 25 (2): 363-78.

[3] Argani P, Ladanyi M, et al.: Renal carcinomas associated with Xp11.2 translocations/TFE3 gene fusions. In: Eble JN, Sauter G, Epstein JI, Sesterhenn IA, editors. Pathology and Genetics of Tumours of the Urinary System and Male Genital Organs (World Health Organization Classification of Tumours) Lyon, France: IARC; 2004; 358 (1): 37-8.

[4] Ellati RT, Abukhiran I, Alqasem K, Jasser J, Khzouz J, Bisharat, et al.: Clinicopathologic features of translocation renal cell carcinoma. Clin Genitourin Cancer, 2017; 15 (1): $112-6$.

[5] Bruder E, Passera O, Harms D, et al.: Morphologic and molecular characterization of renal cell carcinoma in children and young adults. Am J Surg Pathol 2004; 28 (9): 1117-32.

[6] Kinetic A, Jeruc J, et al.: Xp11.2 translocation renal carcinoma in young adults; recently classified distinct subtype. Radiol Oncol 2014; 48 (2): 197-202.

[7] Argani P, Antonescu CR, Illei PB, et al.: Primary renal neoplasms with the ASPL-TFE3 gene fusion of alveolar soft part sarcoma: a distinctive tumor entity previously included among renal cell carcinomas of children and adolescents. Am J Pathol 2001; 159 (1): 179-92.

[8] M. C. Mir, E. Trilla, I. M. De Torres, et al.: Altered transcription factor E3 expression in unclassified adult renal cell carcinoma indicates adverse pathological features and poor outcome. BJU International, 2011; 108 (2): 71-6.

[9] Argani P, Olgac S, Tickoo SK, et al.: Xp11 translocation renal cell carcinoma in adults: expanded clinical, and genetic spec. Am J Surg Pathol 2007; 31 (8): 1149-60.

[10] Kato H, Kanematsu M, Yokoi S, et al.: Renal cell carcinoma associated with Xp11.2 translocation/TFE3 gene fusion: radiological findings mimicking papillary subtype. J Magn Reson Imaging 2011; 33 (1): 217-20.

[11] Chen X, Zhu Q, Li B, et al.: Renal cell carcinoma associated with Xp11.2 translocation/TFE gene fusion: imaging findings in 21 patients. Eur Radiol 2017; 27 (2): 543-52.

[12] Liu K, Xie P, Peng W, et al.: Renal carcinomas associated with Xp11.2translocations/TFE3 gene fusions: findings on MRI and computed tomography imaging. J Magn Reson Imaging 2014; 40 (2): 440-7.

[13] Dey B, Badhe B, Govindarajan KK and Ramesh RA, et al.: $\mathrm{Xp} 11.2$ translocation renal cell carcinoma diagnosed by immunohistochemistry and cytogenetics. J Lab Physicians 2016; 8 (2): 123-5.

[14] Meyer PN, Clark JI, Flanigan RC, et al.: Xp11.2 translocation renal cell carcinoma with a very aggressive course in five adults. Am J Clin Pathol 2007; 128 (1): 70-9.

[15] Armah HB, Parwani AV, Surti U, et al.: Xp11.2 translocation renal cell carcinoma occurring during pregnancy with a novel translocation involving chromosome 19: a case report with review of the literature. Diagn Pathol 2009; 18 (4): 15.

[16] Srigley JR, Delahunt B, Eble JN, E, Epstein JI, Grignon D, et al.: The international society of urological pathology (ISUP) Vancouver classification of renal neoplasia. Am J Surg Pathol 2013; 37 (10): 1469-89.

[17] Green WM, Ionescu R, Morsberger L, Morris K, Netto GJ, et al.: Utilization of a TFE3 break-apart FISH assay in a renal tumor consultation service. Am J Surg Pathol 2013; 37 (8): 1150-63.

[18] Ahluwalia P, Nair B, and Kumar G, et al.: Renal cell carcinoma associated with Xp11.2 translocation/TFE3 gene fusion: A rare case report with review of the literature. Case Rep Urol 2013: 13 (2): 810590.

[19] Song HC, Sun N, Zhang WP, He L, Fu L and Huang C, et al.: Biological characteristics of pediatric renal cell carcinoma associated with Xp11.2 translocations/TFE3 gene fusions. J Pediatr Surg 2014; 49 (4): 539-42.

[20] Kaikki K, Miyata Y, Mochizuki Y, Iwata T, Obatake M, et al.: Long-term treatment with sequential molecular targeted therapy for Xp11.2 translocation renal cell carcinoma: A case report and review of the literature. Clin Genitourin Cancer 2017; 15 (3): 503-6.

[21] Suntour Bonnange H, Leroy X, Chauvet MP, Classe M, et al.: Cutaneous metastases during an aggressive course of Xp11.2 translocation renal cell carcinoma in a teenager. Pediatr Blood Cancer 2014; 61 (9): 1698-700. 
[22] Choueiri TK, Lim ZD, Hirsch MS, et al.: Vascular endothelial growth factor- targeted therapy for treating adult metastatic Xp11.2 translocation renal cell carcinoma. Cancer 2010; 116 (22): 5219-25.

[23] Kinetic A and Jeruc J, et al.: Xp11.2 translocation renal carcinoma in young adults; recently classified distinct subtype. Radiol Oncol 2014; 48 (2): 197-202.

[24] Komai Y, Fujiwara M, Fujii Y, Mukai H, Jones J, et al.: Adult $\mathrm{Xp} 11$ translocation renal cell carcinoma diagnosed by cytogenetics and immunohistochemistry. Clin Cancer Res 2009; 15 (4): 1170-6.

[25] Xu L, Yang R, Gan W, Chen X, Qiu X, Fu K, Huang J, et al.: $\mathrm{Xp11.2}$ translocation renal cell carcinomas in young adults. BMC Urol 2015; 15 (103): 57.

[26] Klatte T, Streubel B, Wrba F, Remzi M, Krammer B, et al.: Renal cell carcinoma associated with transcription factor E3 expression and Xp11.2 translocation: Incidence, characteristics, and prognosis. Am J Clin Pathol 2012; 137 (5): 761-8.

[27] Parwani AV, Surti U, et al.: Xp11.2 translocation renal cell carcinoma occurring during pregnancy with a novel translocation involving chromosome 19: a case report with review of the literature. Diagn Pathol 2009; 4 (1): 15.

[28] Armah HB, Parwani AV, et al.: Xp11.2 translocation renal cell carcinoma. Arch Pathol Lab Med 2010; 134 (1): 124-9.

[29] Argani P, Lal P, Hutchinson B, Lui MY, Reuter VE, et al.: Aberrant nuclear immunoreactivity for TFE3 in neoplasms with TFE3 gene fusions: a sensitive and specific immunohistochemical assay. Am J Surg Pathol 2003; 27 (6): 750-61.

[30] Kuroda N, Mikami S, Pan CC, Cohen RJ, Michal M, et al.: Review of renal carcinoma associated with Xp11.2 translocations/TFE3 gene fusions with focus on pathobiological aspect. Histol Histopathol 2012; 27 (2): 13340 .

[31] Oliver R Rua Fernández, Roberto Cornejo, Miguel Navarro Martín, et al: Renal Cell Carcinoma Associated with Xp11.2 Translocation/TFE3 Gene-fusion: A Long Response to Mammalian Target of Rapamycin (mTOR) Inhibitors. Urology 2018; 117 (1): 41-43.

[32] Bhatt, J. R. \& Finelli, A, et al.: Landmarks in diagnosing and treating renal cell carcinoma. Nat Rev Urol 2014; 11 (9): 517 25 .

[33] Argani, P, Man Yee Lui, Jérôme Couturier, Raymonde Bouvier, et al: A novel CLTC-TFE3 gene fusion in pediatric renal adenocarcinoma with $\mathrm{t}(\mathrm{X} ; 17)(\mathrm{p} 11.2 ; \mathrm{q} 23)$. Oncogene 2003; 22 (34): 5374-8.

[34] Ross H, Argani P, et al.: Xp11 translocation renal cell carcinoma. Pathology 2010; 42 (2): 369-73.

[35] Argani P, Antonescu CR, Couturier J, et al.: PRCC-TFE3 renal carcinomas: morphologic, immunohistochemical, ultrastructural, and molecular analysis entity associated with the t (X;1) (p11.2; q21). Am J Surg Pathology 2002; 26 (12): $1553-66$. 